\title{
Frequency of the Dengue Fever in Patients with Acute Febrile Illness at Diagnostic \& Research Laboratory, Hyderabad
}

\author{
Abid Hussain Chang, Faheem Ahmed Memon, Jai Kirshan, Ameer Bux Detho, \\ Muhammad Muqeem Mangi, Khalid Yousif Memon
}

\begin{abstract}
OBJECTIVE: To determine the frequency of dengue fever in patients with acute febrile illness and to evaluate the clinical profile of dengue positive cases.

METHODOLOGY: This cross sectional, descriptive study was conducted at Diagnostic \& Research Laboratory, LUMHS Hyderabad, Samples of the patients having history of acute febrile illness, within the period of June 2018 to August 2018, were confirmed for the presence of dengue IgM antibody and NS1 antigen. Dengue IgM antibody was detected by using the technique enzyme linked immunosorbent assay (ELISA) while dengue NS1 antigen was detected by immune-chromatographic technique (ICT) rapid device. Clinical and hematological findings were also recorded.

RESULTS: An aggregate of 11250 cases presented with classical characteristics of Dengue fever were collected out of which majority of the cases were in there third decade of life expectancy with median age of 30 years. Seventy four percent $(74 \%)$ i-e 8,325 patients were tested were found to be positive for dengue virus and remaining $2,925(26 \%)$ appeared to be negative for dengue; from which 900 $(8 \%)$ were positive for malarial parasite, $675(6 \%)$ were diagnosed with typhoid fever and strikingly 1350 $(12 \%)$ patients remained undiagnosed.

CONCLUSION: Fever along-with body ache followed by vomiting and abdominal pain were found to be the common characteristic symptoms of Dengue fever.
\end{abstract}

KEY WORDS: Dengue Fever, Aedes Aegypti, IgM, ELISA.

This article may be cited as: Chang AH, Memon FA, Kirshan J, Detho AB, Mangi MM, Memon KY. Frequency of the Dengue Fever in Patients with Acute Febrile Illness at Diagnostic \& Research Laboratory, Hyderabad. J Liaquat Uni Med Health Sci. 2019;18(04): 285-9. doi: 10.22442/jlumhs.191840644

\section{INTRODUCTION}

Dengue fever is a mosquito-borne major viral infection that causes mild to severe illness / disease ${ }^{1}$. Currently, World Health Organization (WHO) estimates 390 million dengue virus contaminations per annum out of which 96 million (67-136 millions) leads to dengue fever ${ }^{2}$. Dengue virus is a single, positive stranded ribonucleic acid (RNA) virus from the family Flaviviridae it has four different serotypes (DEN1-4) and is transmitted to the host by a mosquito vector ${ }^{3}$. Transmission of any one of the four dengue serotypes that are DENV1, DENV2, DENV3 and DENV4 ${ }^{4}$ human beings occurs through the sting of a disease-ridden female Aedes aegypti and less commonly by Aedes albopictus mosquitoes, which feed mostly at dusk and dawn, most frequently two hours after sunrise and few hours before sunset ${ }^{5}$. Noteworthy growths in the mosquito larval populations during the monsoon season may be a reason for the outbreaks of dengue fever ${ }^{6}$. Making its entrance in 1780, Benjamin Rush described this illness as break bone fever, from then Dengue fever (DF) has become as a key community health alarm with clinical spectrum ranging from self-limiting infection to the life frightening hemorrhagic disease.

Clinically, dengue infection can be presented in three different manifestations including classical dengue fever, dengue hemorrhagic fever and dengue shock syndrome. The patients with Classical dengue fever will have abrupt arrival of high-pitched fever, escorted by severe retro-orbital headache, myalgia, arthralgia, nausea, vomiting and macular or maculopapular rash $^{7}$. Presence of hemorrhagic rash or hemorrhagic manifestation in complement to classical DF describes the Dengue Hemorrhagic Fever (DHF), while the dengue shock syndrome is illustrated by hypotension, altered mental status and late capillary filling ${ }^{8}$. Dengue hemorrhagic fever and dengue shock syndrome manifests severe complications and can steer to death $^{9}$. The fatality rate which is high as $10 \%$ can be decreased to as low as $1 \%$ with early recognition and proper treatment. This virus affects about 50-100 million people per annum. The cases of dengue hemorrhagic fever range from 20,000 to 500,000 per annum. 
Thrombocytopenia is a noticeable element of infection, mostly seen within the range $57,000 \pm 5477.07 / \mathrm{LL}^{10}$. A platelet tally below $100 \times 10^{9} / \mathrm{L}$ is a pinpointing measurement for dengue hemorrhagic fever $(\mathrm{DHF})^{11}$. There are multiple cases in which thrombocytopenia occurs including dengue infections but the cause of this remains unknown; mostly thrombocytopenia is due to bone marrow suppression and peripheral platelet destruction. Peripheral immune complex mediated demolition maybe the key factor for thrombocytopenia in dengue infection ${ }^{12}$.

Incidence of dengue fever has increased at an alarming rate in Pakistan and the disease has now become an important public health problem ${ }^{13}$. It is therefore important to examine current demographic trend and clinical profile of DF in the region.

\section{METHODOLOGY}

This cross sectional descriptive study was conducted at Diagnostic \& Research Laboratory, LUMHS Hyderabad, Following all aseptic measures a total of $6 \mathrm{ml}$ whole blood samples were collected by the help of sterilized syringe, from the patients representing acute febrile illness during the period commencing from June 2018 to August 2018. $3 \mathrm{ml}$ blood sample was drawn in vacutainer containing ethylene-di-amine tetraacetic acid (EDTA) as an anticoagulant from which complete blood count indices were measured by XN 10006 parts fully automated analyzer by Sysmex Japan, Leishman Stain peripheral blood smear was prepared to examine the morphology of different cells and to observe the malarial parasites. While $3 \mathrm{ml}$ blood was drawn in plain vacutainer, which was subjected to centrifugation at 5000 RPM for 10 minutes to drawn serum. Which was tested for the presence of dengue IgM antibody and NS1 antigen, using enzyme linked immunosorbent assay (ELISA) and immunochromatographic (ICT) rapid tests respectively completely relying as per manufacturer literature for efficacy $^{14}$. All afebrile patients were not encompassed in the study.

Clinical and hematological outcomes were documented. Basic statistical tools were used for the analysis of data.

\section{RESULTS}

A total of 11,250 patients presenting classical characteristics of Dengue fever were tested. Majority of them were in the third decade of their lives with median age of 30 years (range 1-75 years) with male [7941] to female [3309] ratio was 2.4:1, out of these, $8,325(74 \%)$ patients tested were positive for dengue virus. Remaining 2,925(26\%) appeared Dengue negative, 900(8\%) were positive for malarial parasite,
$675(6 \%)$ were diagnosed with typhoid fever and strikingly $1350(12 \%)$ patients remained undiagnosed (Graph I).

All the patients had fever $(100 \%)$, majority alongwith Body ache (43\%) followed by vomiting $(26 \%)$, abdominal pain (19\%), headache $(6 \%)$, maculopapular rash (4\%) and Bleeding (2\%) (Graph II).

The laboratory result suggested that thrombocytopenia was common in $7,325(88 \%)$, leucopenia in $6,495(78 \%)$ and raised alanine aminotransferase levels in 5,493 (66\%) patients (Graph III).

1,728 patients were admitted in different hospitals of Hyderabad and majority improved clinically with a mortality rate of only $1 \%$.

\section{GRAPH I: GRAPH SHOWING DIAGOSIS OFPATIENTS OTHER THAN DENGUE (DENGUE NEGATIVE PATIENTS)}

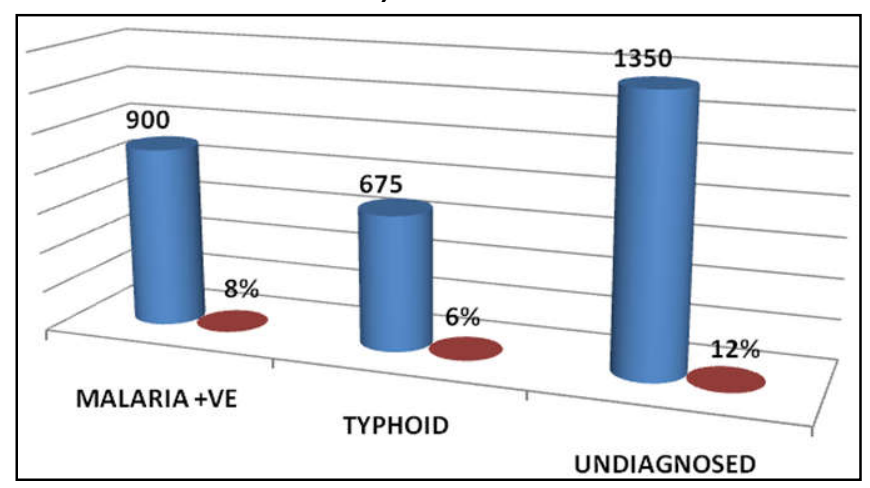

GRAPH II: THE GRAPH SHOWING SIGN AND SYMPTOMS IN DENGUE POSITIVE PATIENTS

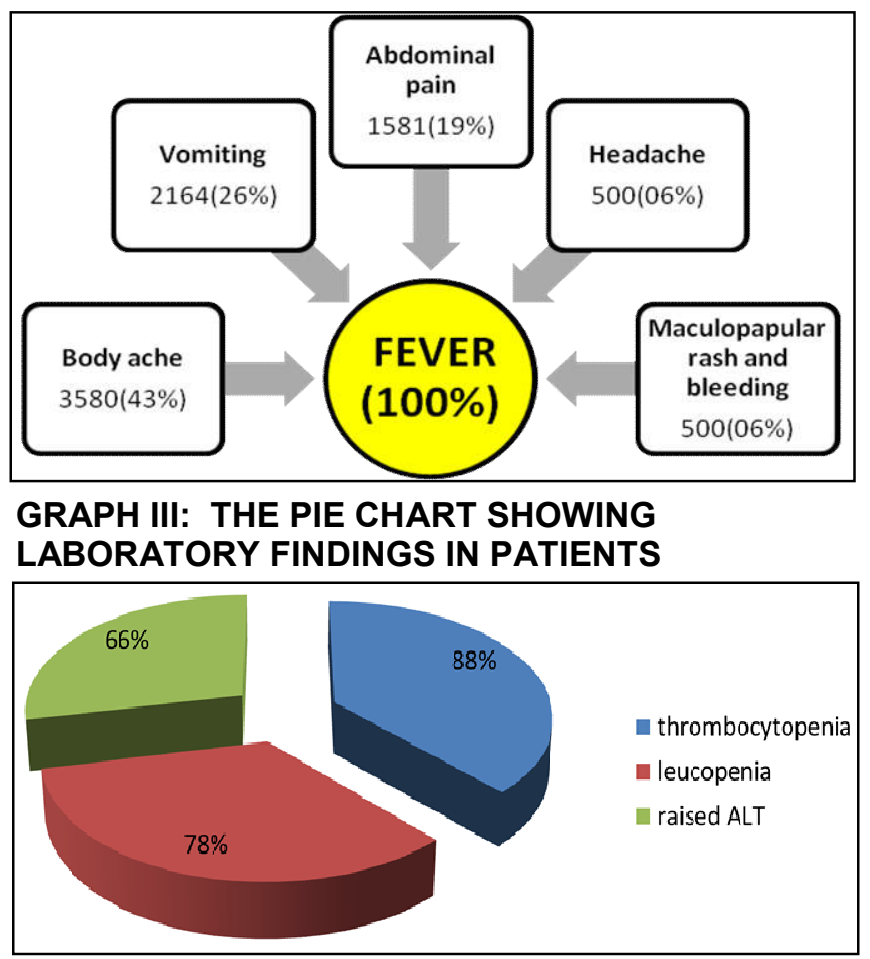




\section{DISCUSSION}

Dengue is developing a serious health hitch for our country. In Pakistan the first reported dengue case was from the metropolitan city of Karachi in 1980's. While Dengue virus has been seen in all the provinces but till 2011 only a few sporadic cases were reported from Peshawar, Haripur, and Abbotabad. The first large-scale epidemic was observed in 2011 in the Province of Punjab, particularly in its largest city of Lahore where more than 21,000 dengue-positive cases and 279 deaths were reported ${ }^{19}$.

The present study was conducted at diagnostic and research laboratory of pathology Department regarding the clinical \& biochemical features of DF cases. The Dengue was so far confirmed by the ELISA that is been considered the most beneficial test for dengue diagnosis, due to its high sensitivity and user friendly procedure. Mostly ELISA is used to detect acute phase (IgM) and convalescent phase (IgG) antibodies, and also for the detection of antigens (Ag). Due to its sensitivity for the detection of acute phase antibodies there is no any requirement for convalescence samples. Since anti-dengue lgM antibodies usually appears within five days of the first clinical symptom. However the IgM production differs significantly between the patients. Some patients will have IgM detectable on the $2^{\text {nd }}$ to $4^{\text {th }}$ day after the commencement of the symptoms, while others do not develop detectable $\operatorname{lgM}$ until the $8^{\text {th }}$ day after the beginning of the disease. The dengue fever is nowadays is considered in the differential diagnosis of fever when it was first expressed. The differential diagnosis associated with DF includes a broad category of viral infections like Çhikungunya and other bacterial, rickettsial \& parasitic infections that results in similar findings. It is impossible to diagnose Dengue infection only on clinical symptoms; therefore a definitive diagnosis requires viral isolation and/or serology 15,16 . The type-specific immunity is life long while hetero type immunity persists for about 2-12 months. In the regions where dengue virus is endemic, cases are more prone to secondary infections with higher incidence of DHF or DSS ${ }^{17}$. In present study, dengue virus was found to be positive in $74 \%$ of the cases, while a study by Gul SN $2014^{20}$ from Lahore shows $98 \%$ positive cases. Cases of DF below 30 years of age were $59 \%$; these findings are consistent with local and international studies from India \& Malaysia. Mostly cases were reported in the months of July, August, September \& October. The main presenting feature, noted in dengue patients was high grade fever $(100 \%)$ of cases which is also noted by Ahmed $S$ et $\mathrm{al}^{8}$ from Karachi. Vomiting was observed in $26 \%$ cases in present study while a study by Khan HMS et $\mathrm{al}^{21}$ mentioned vomiting in $45 \%$ cases. Thrombocytopenia \& leucopenia was recorded as common laboratory findings of DF in our study patients. Platelet compared less than $150 \times 10^{9} / \mathrm{L}$ was seen in $88 \%$ of patients. Riaz MM et $\mathrm{al}^{22}$ described thrombocytopenia in $83 \%$ cases. Leucopenia was reported in $30 \%$ cases by Ahmed S et $\mathrm{al}^{23}$, however in present study it was found in $78 \%$ cases, while a study conducted by Khan $\mathrm{AH} 2010^{7}$ at the same institute shows $80 \%$. Raised liver enzymes were found in $66 \%$ cases in this study population which is in accordance to Soni A $2017^{24}$ In present study $90 \%$ Dengue positive patients showed antigen within $1^{\text {st }}$ week while antibody was found negative because antibody usually develops after 7-8 days of infection, these findings were in accordance with Singh MP et $\mathrm{al}^{25}$ This study highlights that the dengue $\operatorname{lgG}, \lg \mathrm{M}$, IgA and dengue antigen test by ICT method had low sensitivity but high specificity and had a positive predictive value $(0.74$ or $74 \%)$.

\section{CONCLUSION}

Fever along with body ache followed by vomiting and abdominal pain were the common symptoms of Dengue fever. Educating healthcare professionals and public regarding early, appropriate diagnosis is particularly essential to prevent life threatening complications like hemorrhage and shock. A combined methodology is required to reduce the hazard through vector control approaches and improvement of socioeconomic trends ${ }^{18}$.

Ethical Permission: Dean Faculty of Basic Medical Sciences, LUMHS Jamshoro, Letter No. DOC/ LUMHS/DEAN/149 Dated: 11-06-2019.

Conflict of interest: Authors of the study have no conflict of interest to declare.

Funding: There is no Grant or other financial support for this project.

\section{REFERENCES}

1. Rafique I, Saqib MA, Munir MA, Siddiqui S, Malik IA, Rao $M H$, et al. Dengue knowledge and its management practices among physicians of major cities of Pakistan. J Pak Med Assoc. 2015; 65(4): 392-6.

2. Tauseef A, Umar N, Sabir S, Akmal A, Sajjad S, 
Zulfiqar S. Interleukin-10 as a Marker of Disease Progression in Dengue Hemorrhagic Fever. J Coll Physicians Surg Pak. 2016; 26(3): 187-190. doi:03.2016/JCPSP.187190.

3. Awan AK, Ahmed I, Ahmed A, Lone K, Rafique R, Hyderi RM, et al. Clinical Manifestations of Dengue in a Recent Outbreak in Muzaffarabad. Int J Pathol. 2016; 14(4): 148-153.

4. Lemes RP. Comments on the clinical and laboratory characteristics of patients with Dengue Hemorrhagic Fever Manifestations and their transfusion profile. Rev Bras Hematol Hemoter. 2014; 36(2): 100-1. doi:10.5581/1516-8484. 20140022.

5. Khaskheli A, Qazi N, Harani M, Ujjan I. Pattern of Dengue Virus Infection Cases Admitted In Tertiary Care Hospital at Hyderabad, Sindh. J Liaquat Uni Med Health Sci. 2010; 09(03): 142-4.

6. Thavara U, Tawatsin A, Chansang C, Kong-ngamsuk W, Paosriwong S, Boon-Long J, et al. Larval occurrence, oviposition behavior and biting activity of potential mosquito vectors of dengue on Samui Island, Thailand. J Vector Ecol. 2001; 26(2): 172-80.

7. Khan AH, Hayat AS, Masood N, Solangi NM, Shaikh TZ. Frequency and Clinical Presentation of Dengue Fever at Tertiary Care Hospital of Hyderabad/Jamshoro. J Liaquat Uni Med Health Sci. 2010; 09(02): 88-94.

8. Ahmed S, Ali N, Ashraf S, llyas M, Tariq WU, Chotani RA. Dengue Fever Outbreak: A Clinical Management Experience. J Coll Physicians Surg Pak. 2008; 18(1): 8-12. doi:01.2008/JCPSP.0812.

9. Rauf A, Shah Kazmi NH, Zaman H, Gillani S, Shah T, Malik F, Ismaeel S. Pattern of presentation among adults hospitalized with dengue disease. J Ayub Med Coll Abbottabad. 2017; 29(3): 432-35.

10. Fida Z, Khan Z, Khan A, Khan H, Ur Rehman F. Outcome of Patients with Dengue Fever. Gomal J Med Sci. 2016; 14(3): 137-40.

11. Nimmannitya S. Clinical spectrum and management of dengue haemorrhagic fever. Southeast. Southeast Asian J Trop Med Public Health. 1987; 18(3): 392-7.

12. Hassan FS, Akhtar R, Mustafa MA, Kazmi SU, Nadeem M. To Evaluate the Efficacy and Reliability of commonly available tests for the diagnosis of Dengue Fever in Children in Karachi. Pak J Med Res. 2013; 52(3): 84-7.
13. Qureshi EMA, Tabinda AB, Vehra $S$. Sero-surveillance of dengue in the city Lahore, Pakistan JPMA. 2017; 67(8): 1173-9.

14. Lutfullah G, Ahmed J, Khan A, Ihsan H, Ahmed J. Evaluation of Non-Structural Protein-1(NS1) positive patients of 2013 dengue outbreak in Khyber Pakhtunkhwa, Pakistan. Pak J Med Sci. 2017; 33(1): 172-6.

15. Chadwick D, Arch B, Wilder-Smith A, Paton N. Distinguishing dengue fever from other infections on the basis of simple clinical and laboratory features: application of logistic regression analysis. J Clin Virol. 2006; 35(2): 147-53.

16. Martinez-Vega RA, Diaz-Quijano FA, Villar-Centeno LA. Low concordance between early clinical suspicion of dengue and its serological confirmation. Rev Med Chil. 2006; 134 (9): 1153-60.

17. Chaudhary R, Khetan D, Sinha S, Sonker A, Pandey P, Das SS, et al. Transfusion support to dengue patients in a hospital based blood transfusion service in north India. Transfus Apher Sci. 2006; 35(3): 239-44.

18. Raza FA, Ashraf S, Hasnain S, Ahmad J, Iqbal M. Dengue seroprevalence and its socioeconomic determinants in Faisalabad, Pakistan: a cross-sectional study. Rev Soc Bras Med Trop. 2018; 51(4): 503-7.

19. Ahmed Qureshi EM, Tabinda AB, Vehra S. Predicting dengue outbreak in the metropolitan city Lahore, Pakistan, using Dengue vector indices and selected climatological variables as predictors. J Pak Med Assoc. 2017; 67(3): 41621.

20. Gul SN, Ghafoor F, Jajja MA. Knowledge, Attitude and Practices Regarding Dengue Fever in Lahore, Pakistan. Pak J Med Res. 2014; 53(2): 34-8.

21. Khan HMS, Zaman Q, Khan BA, Arif M, Raza A, Haq A. Retrospective Analysis of 68 Cases of Dengue Fever. Pak J Med Res. 2012; 51(1): 18-20.

22. Riaz MM, Mumtaz K, Khan MS, Patel J, Tariq M, Hilal $\mathrm{H}$, et al. Outbreak of dengue fever in Karachi 2006: A clinical perspective. J Pak Med Assoc. 2009; 59(6): 339-44.

23. Ahmed S, Arif F, Yahya Y, Rehman A, Abbas K, Ashraf $S$, et al. Dengue fever outbreak in Karachi 2006 - A study of profile and outcome of children under 15 years of age. J Pak Med Assoc. 2008; 
58(1): 4-8.

24. Soni A, Patel PM, Malhi NS, Avasthi GL. Spectrum of liver dysfunction in patients with dengue infection and the markers of severe disease: study from a tertiary care centre in Punjab. J Liver Res Disord Ther. 2017; 3(4): 95-8. doi:10.15406/jlrdt.2017.03.00063.

25. Singh MP, Majumdar M, Singh G, Goyal K, Preet $\mathrm{K}$, Sarwal A, et al. NS1 antigen as an early diagnostic marker in dengue: report from India. Diagn Microbiol Infect Dis. 2010; 68(1): 50-4. doi:10.1016/j.diagmicrobio.2010.04.004.

\begin{tabular}{|l|}
\hline AUTHOR AFFILIATION: \\
Dr. Abid Hussian Chang (Corresponding Author) \\
Associate Professor, Department of Pathology \\
Liaquat University of Medical \& Health Sciences \\
(LUMHS), Jamshoro, Sindh-Pakistan. \\
Email: drabidchang88@yahoo.com \\
Dr. Faheem Ahmed Memon \\
Lecturer, Department of Pathology \\
LUMHS, Jamshoro, Sindh-Pakistan. \\
Dr. Jai Kirshan \\
Associate Professor, Department of Pathology \\
CMC/ SMBBU Larkana, Sindh-Pakistan. \\
Dr. Ameer Bux Detho \\
Assistant Professor, Department of Pathology \\
PUMHS Nawabshah (SBA), Sindh-Pakistan. \\
Dr. Muhammad Muqeem Mangi \\
Assistant Professor, Department of Physiology \\
GMMMC Sukkur, Sindh-Pakistan. \\
Dr. Khalid Yousif Memon \\
Lecturer, Department of Pathology \\
LUMHS, Jamshoro, Sindh-Pakistan. \\
\hline
\end{tabular}

